\title{
Precise test of Higgs boson properties via triple Higgs boson production in vector boson fusion at future colliders
}

\author{
A. S. Belyaev, ${ }^{1,2, *}$ P. B. Schaefers, ${ }^{1, \dagger}$ and M. C. Thomas ${ }^{1, *}$ \\ ${ }^{1}$ School of Physics \& Astronomy, University of Southampton, Southampton SO17 1BJ, United Kingdom \\ ${ }^{2}$ Particle Physics Department, Rutherford Appleton Laboratory, \\ Chilton, Didcot, Oxon OX11 OQX, United Kingdom
}

(Received 9 February 2018; published 23 January 2019)

\begin{abstract}
For certain classes of beyond the standard model theories, including composite Higgs models, the coupling of the Higgs to gauge bosons can be different from the standard model one. In this case, the multiboson production via vector boson fusion (VBF) can be hugely enhanced in comparison to the SM production one due to the lack of cancellation in longitudinal vector boson scattering. Among these processes, triple Higgs boson production in VBF plays a special role-its enhancement is especially spectacular due to the absence of background from transversely polarized vector bosons in the final state. While the rates from $p p \rightarrow j j h h h$ production in vector boson fusion are too low at the LHC and even at future $33 \mathrm{TeV} p p$ colliders, we have found that the $100 \mathrm{TeV} p p$ future circular collider (FCC) has the unique opportunity to probe the $h V V$ coupling far beyond the LHC sensitivity. We have evaluated the $p p \rightarrow j j h h h$ rates as a function of deviation from the $h V V$ coupling and have found that the background is much smaller than the signal for observable signal rates. We also found that the $100 \mathrm{TeV} p p$ FCC can probe the $h V V$ coupling up to the permille level, which is far beyond the LHC reach. These results highlight a special role of the $h h h \mathrm{VBF}$ production and stress once more the importance of the $100 \mathrm{TeV} p p$ FCC.
\end{abstract}

DOI: 10.1103/PhysRevD.99.015030

\section{INTRODUCTION}

In 2012, a new scalar particle was discovered during the first run of the LHC with a collision energy of $\sqrt{s}=8 \mathrm{TeV}$ $[1,2]$. Although the found particle is thought to fit the standard model (SM) Higgs boson astonishingly well, it is still possible that it belongs to a different theory such as a composite Higgs model, supersymmetry or some other theory.

The increase of LHC energy and luminosity as happened in LHC Run 2 has allowed to understand Higgs boson properties more precisely. The status of the Higgs properties reported by ATLAS and CMS collaborations [3,4] for $80 \mathrm{fb}^{-1}$ integrated luminosity, indicates the current LHC sensitivity to the $h W W, h Z Z, h \gamma \gamma$ and $h g g$ couplings at about $10 \%$ level and Higgs couplings to the third generation of fermions at the 15-25\% level. At high luminocity LHC (HL-LC) we expect the sensitivity to Higgs boson

\footnotetext{
*A.Belyaev@soton.ac.uk

†P.Schaefers@ soton.ac.uk

"M.C.Thomas@soton.ac.uk
}

Published by the American Physical Society under the terms of the Creative Commons Attribution 4.0 International license. Further distribution of this work must maintain attribution to the author(s) and the published article's title, journal citation, and DOI. Funded by SCOAP ${ }^{3}$. coupling to gauge bosons and fermions to be increased to the level of few (3-5) percent (see e.g., [5,6]). This accuracy, however is not sufficient to probe a broad range of theories including supersymmetry which predicts deviation of the Higgs couplings from the SM at the percent level or below (see e.g., [7-14] and the references therein). The future colliders such as $33 \mathrm{TeV}$ LHC, $350 \mathrm{GeV} e^{+} e^{-}$future circular collider (FCC-ee) [15] $100 \mathrm{TeV} p p$ future circular collider (FCC-hh) $[16,17]$ or 0.25-1 TeV International Linear Collider (ILC) [18] has potential to significantly improve the measurement of the Higgs boson properties. For example, $h W W$ and $h Z Z$, couplings are expected to be measured with the accuracy of about $0.3-0.5 \%$ at ILC and at similar level at FCC.

One should note that while the measurement of the Higgs self-couplings (triple, $\lambda_{3}$ and quartic, $\lambda_{4}$ ones) are problematic even at HL-LHC, $\lambda_{3}$ can be measured with $3 \%$ accuracy at the FCC-hh $[16,17]$ and $\lambda_{4}$ coupling, as it was shown in Ref. [19], can be confirmed at $3 \sigma$ level with $\tau \tau b b b b$ process from triple Higgs boson production in gluon fusion process (also see CERN Yellow Book [20] for complete set of references). Further progress in this direction has been done in [21].

In this work we present novel results of using triple Higgs boson production from vector boson fusion process in order to probe Higgs boson coupling to vector bosons, $h V V$, with unique precision at $100 \mathrm{TeV}$ FCC-hh collider. 
As a case study, we consider an effective field theory (EFT) based on a nonlinear $\sigma$ model $(\mathrm{NL} \sigma \mathrm{M})$, where the Higgs boson arises as a field expansion in the EFT. The corresponding Higgs couplings to itself and the gauge bosons thus can be described by their SM couplings modified by some multiplicative parameters and might very well take non-SM values. As a consequence, the vector boson scattering can be highly enhanced in such classes of models due to the lack of unitarity cancellations at high energies. We investigate such an effect with the focus on triple Higgs boson production in vector boson fusion process (VBF) at high energy future proton-proton colliders. It was shown previously that triple Higgs boson production in VBF is especially interesting, since its cross section increases considerably faster (in comparison to the SM) than for other processes with two or three vector bosons in the final states [22]. We have found that VBF triple Higgs boson production can only be visible at the $100 \mathrm{TeV} p p$ FCC, however, the potential of this collider to explore the $h V V$ coupling to vector bosons via this process is impressive: the process is effectively free of background for the boosted triple Higgs signature and at high luminosity, the $h V V$ coupling can be measured up to permille precision.

The paper is organized as follows. In Sec. II, we discuss the nonlinear $\sigma$ model and unitarity as well as the cross section enhancement for multiboson production in vector boson scattering at high energies. In Sec. III, we present results for the signal rates and distributions at the LHC and future $p p$ colliders. In Sec. IV, we estimate the background for the VBF triple Higgs boson signal and find the potential of the $100 \mathrm{TeV} p p$ FCC to measure the $h V V$ coupling. Finally, we draw our conclusions in Sec. V.

\section{UNITARITY AND THE NONLINEAR $\sigma$ MODEL}

In particle physics, one important quantity to describe particle scatterings are their cross sections, and high cross sections mean more likely detections of such scatterings. However, cross sections cannot grow arbitrarily large and are limited by an upper bound, the unitarity bound. For a $2 \rightarrow n$ scattering with collision energy $s$, the unitarity bound takes on the form $[23,24]$

$$
\sigma(2 \rightarrow n)<\frac{4 \pi}{s} .
$$

The most general cross section for a $2 \rightarrow n$ scattering is proportional to

$$
\sigma(2 \rightarrow n) \sim \frac{1}{s} \mathcal{A}^{2}(s) s^{n-2},
$$

where $\frac{1}{s}$ corresponds to the flux factor, $\mathcal{A}^{2}(s)$ is the squared scattering amplitude and $s^{n-2}$ gives the energy dependence of the phase space integral $[23,25]$

$$
\begin{aligned}
R_{n}(s) & =\int \prod_{i=1}^{n} \frac{\mathrm{d}^{3} p_{i}}{(2 \pi)^{3}\left(2 E_{i}\right)^{3}}(2 \pi)^{4} \delta^{4}\left(\sqrt{s}-\sum_{i=1}^{n} p_{i}\right) \\
& =\frac{(2 \pi)^{4-3 n}\left(\frac{\pi}{2}\right)^{n-1}}{(n-1) !(n-2) !} s^{n-2}
\end{aligned}
$$

for massless particles in four dimensions. Together with Eq. (1), this restricts the scattering amplitude $\mathcal{A}$ to be proportional to

$$
\mathcal{A}(2 \rightarrow n) \sim s^{1-\frac{n}{2}}
$$

in order for unitarity to be fulfilled.

So far, all considerations have been model-independent, although it turns out that Eq. (4) is true for the SM, if the theory contains a Higgs boson. This feature of the SM amplitude is special and not generic for other models. Consider the following Lagrangian of a nonlinear $\sigma$ model $(\mathrm{NL} \sigma \mathrm{M})$

$$
\mathcal{L}_{\mathrm{NL} \sigma \mathrm{M}}=\frac{v^{2}}{4} \operatorname{Tr}\left[\partial_{\mu} U \partial^{\mu} U^{\dagger}\right],
$$

where $v=246 \mathrm{GeV}$ is the usual scale of electroweak symmetry breaking and

$$
U=\mathrm{e}^{\mathrm{i} \cdot \vec{r} \cdot \vec{\pi}} .
$$

with $\vec{\pi}$ being the massless Goldstone bosons of the theory. By using the equivalence theorem [26-31], these can be identified with the longitudinal vector bosons in the high energy limit.

One can show by naïve power counting that the scattering amplitudes in the $\mathrm{NL} \sigma \mathrm{M}$ grow lienarly in $s$, i.e.,

$$
\mathcal{A}_{\mathrm{NL} \sigma \mathrm{M}}(2 \rightarrow n) \sim \frac{s}{v^{n}} .
$$

As a consequence, the cross sections grow arbitrarily large and unitarity is violated for any scattering process in the $\mathrm{NL} \sigma \mathrm{M}$. In order to restore unitarity, the model must be repaired in the UV region, where unitarity violation occurs. ${ }^{1}$ This can be achieved by adding a scalar field, call it the Higgs field, to the model, coupling to the lightest degrees of freedom. This is similar to the case in the SM, where the Higgs is mandatory to cancel unitarity-violating contributions in longitudinal vector boson scattering $\left(W_{L} W_{L} \rightarrow W_{L} W_{L}\right)$.

It is convenient to describe the NL $\sigma \mathrm{M}$ together with the Higgs field in terms of an EFT, where we expand operators around the Higgs field. The corresponding Lagrangian takes the following form [32]

\footnotetext{
${ }^{1}$ When the UV region starts varies and depends mainly on how many particles are produced in the final state and how big $S$ is [22].
} 


$$
\begin{aligned}
\mathcal{L}_{\text {eff }}= & \frac{v^{2}}{4}\left(1+2 a \frac{h}{v}+b \frac{h^{2}}{v^{2}}+b_{3} \frac{h^{3}}{v^{3}}+\cdots\right) \operatorname{Tr}\left[\partial_{\mu} U \partial^{\mu} U^{\dagger}\right] \\
& +\frac{1}{2}\left(\partial_{\mu} h\right)^{2}-\frac{1}{2} m_{h}^{2} h^{2}-d_{3} \lambda v h^{3}-d_{4} \frac{\lambda}{4} h^{4}+\cdots,
\end{aligned}
$$

where $a, b, b_{3}, d_{3}, d_{4}$ are dimensionless parameters changing the overall coupling strength of a certain term. By setting $a=b=d_{3}=d_{4}=1, b_{3}=0$ and redefining the Higgs, the SM is restored and there is no unitarity violation. On the other hand, changing these parameters will lead to large increases in cross sections at high scattering energies along with unitarity violation, since the cancellations mentioned earlier cannot be fully compensated for by the Higgs. The energy scale at which unitarity violation starts to appear therefore is the upper limit for the validity of an EFT. Beyond this scale, the EFT is no longer a good approximation of nature and a new model or modifications to the old one are needed. In either case, this behavior can be used as an indicator of new physics.

In this work, we choose $b=d_{3}=d_{4}=1$ and $b_{3}=0$, but leave $a$ as a free parameter. In other words, we consider the SM with a modified coupling between one Higgs and two gauge bosons.

In the next section, we revisit and update the cross sections for different scattering processes involving the modified couplings, as previously studied in Ref. [22].

\section{TRIPLE HIGGS BOSON PRODUCTION VIA VBF}

\section{A. Cross sections for multiple vector boson and Higgs production with two jets}

In order to estimate which process benefits most of the changed couplings in the EFT, we investigate the process $p p \rightarrow j j+X$ with the final states $X$ being either $W^{+} W^{-}$, $W^{+} W^{-} h, h h$ or $h h h$. We compute the regular SM cross sections $(a=1)$ and the cross sections for $a=0.9$. We further compute these cross sections with applied vector boson fusion (VBF) cuts in order to enhance the actual Higgs signal. The cross sections are computed using MADGRAPH5_AMCNLO 2.2.3 [33]. The parton density function (PDF) we use is CTEQ611 [34]. To avoid soft and collinear jets, we assign a general minimum transverse momentum of the jets to be $p_{\mathrm{T}}^{j} \geq 50 \mathrm{GeV}$ and the minimal distance between two jets is set to $\Delta R(j, j) \geq 0.4$. The proton and jet particle content is set to $(p, j)=$ $g, u, \bar{u}, d, \bar{d}, s, \bar{s}, c, \bar{c}$. We have evaluated the complete set of tree-level diagrams for $p p \rightarrow j j h h h$ process including $g g h$ effective vertex, contributing to the $g g \rightarrow h$ fusion process, contribution from which is however became negligible after we apply VBF cuts. The VBF cuts we choose are listed in Table I. Finally, the computed cross sections are shown in Table II.

The first thing to notice is that all cross sections increase with energy. In the SM case $(a=1)$, all cross sections
TABLE I. Parameter values with and without VBF cuts.

\begin{tabular}{lcc}
\hline \hline Parameter & Without VBF cuts & With VBF cuts \\
\hline$E_{j}[\mathrm{GeV}]$ & 0 & 1500 \\
$\Delta \eta$ & 0 & 5 \\
\hline \hline
\end{tabular}

roughly grow by two to three orders of magnitude, if $\sqrt{s}$ is increased from $13 \mathrm{TeV}$ to $100 \mathrm{TeV}$. This is also true if VBF cuts are applied, albeit the impact of the cuts is very different for different processes. For the first two processes with $W^{+} W^{-}$in the final state, VBF cuts will reduce the cross sections by 2 to 3 orders of magnitude, whereas in case of pure Higgs production channels, the cross sections decreases by a factor of around 30 for $13 \mathrm{TeV}$ and by only a factor around 3 for $100 \mathrm{TeV}$ collider. The reason for this is that the processes with only Higgs bosons and jets in the final state are mainly produced through $\mathrm{VBF}$, whereas the processes with $W^{ \pm}$pairs in the final states can be produced through a variety of different channels (e.g., radiation from jets).

Coming now to the non-SM case $(a=0.9)$, triple Higgs production clearly stands out compared to the other processes. Not only it is least affected by VBF cuts (the cross sections decrease by a factor of 12 at $13 \mathrm{TeV}, 1.8$ at $33 \mathrm{TeV}$ and 1.1 at $100 \mathrm{TeV}$ ), but it is also the most significantly enhanced by the change from $a=1$ to $a=0.9$. At $13 \mathrm{TeV}$, the cross section after $\mathrm{VBF}$ cuts increases by a factor of almost 400, whereas at $100 \mathrm{TeV}$, the cross sections is almost $10^{4}$ times larger compared to its SM value. All other processes gain or lose only a negligible part of their SM cross sections. This can be explained by a transversal "pollution" of the cross sections, which is highly present in the non-Higgs processes. Here, the transversal contribution to the cross sections is significantly larger compared to the longitudinal part. This also explains why there is no gain in cross section when moving from $a=1$ to $a=0.9$ for $W^{+} W^{-}$production, as there are only very few diagrams actually involve a coupling of two $W^{ \pm}$to a longitudinal W-bosons.

To summarize the properties of the processes discussed above, it becomes apparent that triple Higgs production offers great potential to explore Higgs properties such as its couplings to other bosons and itself. Due to the huge increase in cross sections (and possible unitarity violations) in the non-SM case, it may also serve as a great tool to explore physics beyond the SM close to the cutoff energy scale of the underlying EFT, as was discussed in Chap. 2. One may not forget, however, that the cross sections for triple Higgs production after all are still only in the range of several $\mathrm{fb}$ and small compared to cross sections other processes can achieve. For this reason, it is also important to investigate the possible backgrounds for triple Higgs production, estimate the signal-to-background ratio and a signal significance for a given luminosity. This analysis is performed in chapter 4 . 
TABLE II. Cross sections in pb for different processes with variable $a, \sqrt{s}$ and VBF cuts. The cross $(\times)$ indicates the cross sections before VBF cuts, while the tick $(\checkmark)$ refers to the cross sections after VBF cuts.

\begin{tabular}{|c|c|c|c|c|c|c|c|}
\hline \multirow[b]{2}{*}{ Process } & \multirow[b]{2}{*}{ VBF cuts } & \multicolumn{2}{|c|}{$13 \mathrm{TeV}$} & \multicolumn{2}{|c|}{$33 \mathrm{TeV}$} & \multicolumn{2}{|c|}{$100 \mathrm{TeV}$} \\
\hline & & $a=1.0$ & $a=0.9$ & $a=1.0$ & $a=0.9$ & $a=1.0$ & $a=0.9$ \\
\hline$p p \rightarrow j j W^{+} W^{-}$ & $\begin{array}{l}x \\
y\end{array}$ & $\begin{array}{c}9.88 \\
1.29 \times 10^{-2}\end{array}$ & $\begin{array}{c}9.88 \\
1.27 \times 10^{-2}\end{array}$ & $\begin{array}{c}60.56 \\
0.48\end{array}$ & $\begin{array}{c}60.48 \\
0.47\end{array}$ & $\begin{array}{c}352.14 \\
5.49\end{array}$ & $\begin{array}{c}352.49 \\
5.47\end{array}$ \\
\hline$p p \rightarrow j j W^{+} W^{-} h$ & $\begin{array}{l}x \\
\checkmark\end{array}$ & $\begin{array}{l}1.71 \times 10^{-3} \\
1.26 \times 10^{-5}\end{array}$ & $\begin{array}{l}1.43 \times 10^{-3} \\
1.35 \times 10^{-5}\end{array}$ & $\begin{array}{l}1.63 \times 10^{-2} \\
9.30 \times 10^{-4}\end{array}$ & $\begin{array}{l}1.53 \times 10^{-3} \\
1.05 \times 10^{-3}\end{array}$ & $\begin{array}{l}0.69 \\
0.15\end{array}$ & $\begin{array}{l}0.60 \\
0.19\end{array}$ \\
\hline$p p \rightarrow j j h h$ & $\begin{array}{l}x \\
\checkmark\end{array}$ & $\begin{array}{l}5.11 \times 10^{-4} \\
2.13 \times 10^{-5}\end{array}$ & $\begin{array}{l}3.64 \times 10^{-4} \\
1.32 \times 10^{-5}\end{array}$ & $\begin{array}{l}3.49 \times 10^{-3} \\
7.65 \times 10^{-4}\end{array}$ & $\begin{array}{l}2.93 \times 10^{-3} \\
7.69 \times 10^{-4}\end{array}$ & $\begin{array}{l}1.70 \times 10^{-2} \\
5.56 \times 10^{-3}\end{array}$ & $\begin{array}{l}1.92 \times 10^{-2} \\
9.20 \times 10^{-3}\end{array}$ \\
\hline$p p \rightarrow j j h h h$ & $\begin{array}{l}x \\
\checkmark\end{array}$ & $\begin{array}{l}2.38 \times 10^{-7} \\
6.14 \times 10^{-9}\end{array}$ & $\begin{array}{l}2.50 \times 10^{-5} \\
2.06 \times 10^{-6}\end{array}$ & $\begin{array}{l}1.97 \times 10^{-6} \\
4.39 \times 10^{-7}\end{array}$ & $\begin{array}{l}1.37 \times 10^{-3} \\
7.48 \times 10^{-4}\end{array}$ & $\begin{array}{l}1.23 \times 10^{-5} \\
4.70 \times 10^{-6}\end{array}$ & $\begin{array}{l}4.60 \times 10^{-2} \\
4.10 \times 10^{-2}\end{array}$ \\
\hline
\end{tabular}

One should note that contribution from $g g \rightarrow h$ process is negligible apply VBF cuts as can be seen from Table II. For $100 \mathrm{TeV}$ collider the $\epsilon_{a}=1-a=0.1$ enhances the SM cross section by 4 orders of magnitude coming from $\mathrm{VBF}$ fusion and not from $g g$ fusion. Even for $\epsilon_{a}=0.001-$ the limit of the $100 \mathrm{TeV}$ FCC-hh we discuss here, the enhancement is about factor of 100 for the VBF in comparison to the SM prediction, so $g g$ is still negligible in comparison to VBF even for that small value of $\epsilon_{a}$.

In the following part, we focus solely on triple Higgs production with applied VBF cuts, and study the impact of the anomalous Higgs coupling $a$ for different collision energies and unitarity bounds.

\section{B. Vector boson scattering level and unitarity}

In Fig. 1 we present a schematic diagram for triple Higgs production, which represents the process under study and the around a hundred actual Feynman diagrams behind it. Before calculating the cross sections for the full hadronic process, however, it is worth investigating only the VBF part of this process, i.e., $V V \rightarrow h h h$ with $V=Z, W^{ \pm}$. In this case, the invariant mass of the three Higgs bosons $M_{h h h}$ is equal to the $V V$ center-of-mass $(\mathrm{CM})$ energy $\sqrt{\hat{s}_{\mathrm{VBF}}}$, so

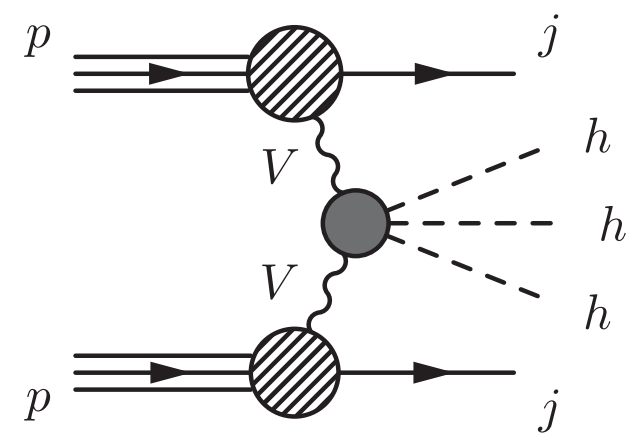

FIG. 1. Schematic diagram for triple Higgs production in VBF. The grey blob in the centre represents many Feynamn diagrams and topologies for two vector-bosons $V=Z, W^{ \pm}$fusion into three Higgs bosons $h$.

$$
M_{h h h}=\sqrt{\hat{s}_{\mathrm{VBF}}}
$$

This relation is very useful in two ways. First, it can be used to calculate the unitarity bound at the VBF stage with high precision. This is achieved by plugging in the cross sections for $V V \rightarrow h h h, \sigma_{V V \rightarrow V V h h h} \equiv \hat{\sigma}(h h h)$, in Eq. (1) and solving for $\sqrt{\hat{s}_{\mathrm{VBF}}}$, which now marks the CM energy, where unitarity is violated. Second, it acts as a link between the level of $V V$ scattering and $q q$ scattering. So if parts of this distribution exceed the unitarity bound found in $\sqrt{\hat{s}_{\mathrm{VBF}}}$, this clearly indicates the presence of new physics, in particular some resonances which should unitarize the scattering amplitude.

In order to address the first point, we computed the cross sections for $V V \rightarrow h h h$ and its dependence on $a$ using CALCHEP 3.6.23 [35]. Figure 2 shows a series of these cross sections for different values of $a$ together with the unitarity bound [Eq. (1)]. The colored curves show the cross sections as functions of $\sqrt{\hat{s}_{\mathrm{VBF}}}$, where dashed lines refer to $a<1$

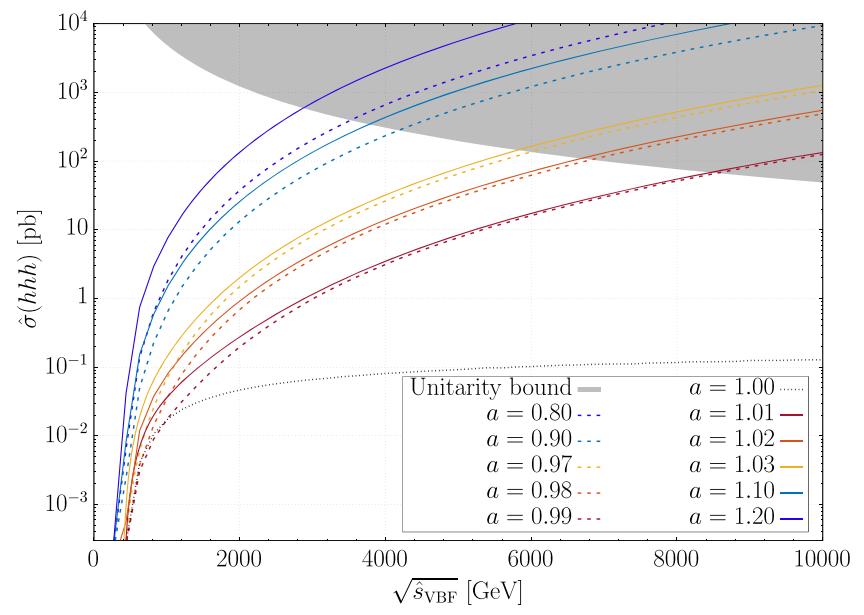

FIG. 2. Cross sections $\hat{\sigma}(h h h)$ in pb for vector boson scattering into three Higgs, $V V \rightarrow h h h, V=Z, W^{ \pm}$, for different values of $a$. The grey area marks the region where unitarity is violated. 
and the solid curves show the cross sections with $a>1$. The dotted black line at the bottom shows the SM cross section for comparison and the grey area in the top right corner marks the region where unitarity is violated. One can observe a huge increase of the cross section for any value of $a \neq 1$ compared to the SM, as discussed in Sec. II. For the shown range of $a \neq 1$, unitarity is violated roughly between $\sqrt{\hat{s}_{\mathrm{VBF}}}=2.4 \mathrm{TeV}$ and $8.4 \mathrm{TeV}$ for $|a-1|$ range between 0.2 and 0.01 respectively. Eventually for $a=1$ unitarity is not violated, since there is no unitarity violation in the SM.

\section{Differential distributions}

In the last chapter, we have seen that triple Higgs production is greatly enhanced when the $h V V$ coupling deviates from the SM one even at the percent level. In this section we take a closer look at the $p p \rightarrow j j h h h$, $\sigma_{p p \rightarrow j j h h h} \equiv \sigma(h h h)$ cross section as a function of anomalous $h V V$ coupling $a$, unitarity violation and differential distribution of the $h h h$ invariant mass. For this purpose using MADGRAPH5_AMCNLO 2.2.3 we computed the total cross section for $p p \rightarrow j j h h h, \sigma_{p p \rightarrow j j h h h} \equiv \sigma(h h h)$ with vector-boson fusion (VBF) cuts applied as a function of $a$ parameter. The results are shown in Fig. 3. For $a=1$, the SM coupling is restored and therefore also the SM cross section. However, even for a small deviation of $a$ from one e.g., for $a=0.98$, the cross sections increase by more than one order of magnitude for $\sqrt{s}=13 \mathrm{TeV}$, by more than two orders of magnitude for $\sqrt{s}=33 \mathrm{TeV}$ and by about three orders of magnitude for $\sqrt{s}=100 \mathrm{TeV}$. If $a$ deviates roughly $10 \%$ from 1 , the increase starts to slow down. For even smaller values of $a$ the cross section reaches extremum at $a \approx 0.6$ and by even further reducing $a$, the cross sections starts to decrease again. On the other side, increasing $a$ beyond 1 will lead only to ever growing cross sections, as the multiplicative nature of $a$ in the coupling starts to dominate the cross sections slope. This behavior has been studied and explained in [22] at the level of $W W$ scattering and is well reproduced here at the level of $\mathrm{pp}$ collisions.

In order to compare the cross sections for different $\sqrt{s}$ and to see the actual gain in cross section compared to the $\mathrm{SM}$, it is useful to normalize the data of Fig. 3 with respect to the SM cross section $\sigma(a=1)$. The resulting cross sections are shown in Fig. 4. Again, the cross section increases fastest in the area $a \in[0.9,1.1]$. This huge enhancement in cross section however comes with the price of (partially) losing unitarity, since there is no exact Higgs cancellation in the VBF channel any longer. As this loss of unitarity indicates where new physics must appear, it is important to know at which energies unitarity is violated and how distinct the violation is.

Since the unitarity violating energy scales of $\sqrt{\hat{s}_{\mathrm{VBF}}}$ are known, we can apply this knowledge for $p p \rightarrow j j h h h$ at
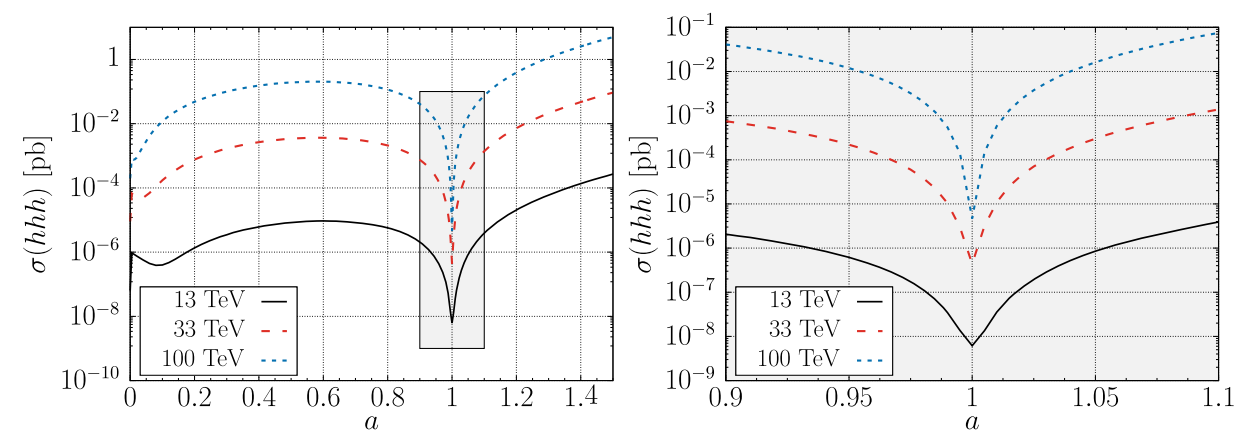

FIG. 3. Cross sections $\sigma(h h h)$ in pb for $p p \rightarrow j j h h h$ with VBF cuts for $\sqrt{s}=13,33,100 \mathrm{TeV}$ in dependance of $a$. The right plot shows a zoomed version of the grey highlighted segment with $a \in[0.9,1.1]$ in the left plot.
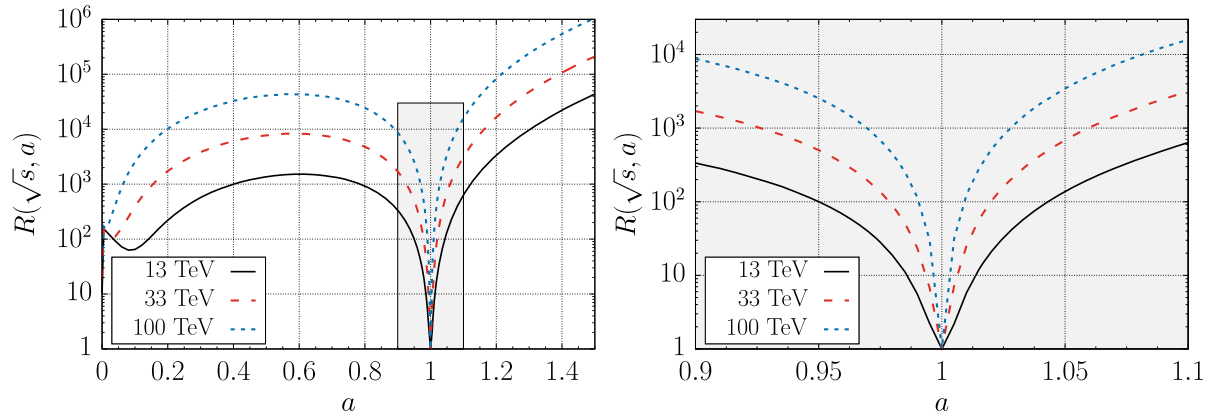

FIG. 4. Ratio $R_{\sqrt{s}}(a)=\frac{\sigma^{p \rightarrow j j h h h}(a)}{\sigma^{p \rightarrow j j h h}(a=1)}$ for $p p \rightarrow j j h h h$ with the data of Fig. $3 . R_{\sqrt{s}}(a)=1$ corresponds to the unmodified SM cross sections. The right plot shows a zoomed version of the grey highlighted segment with $a \in[0.9,1.1]$ in the left plot. 
the level of pp collisions. To do so, we computed the full invariant mass $M_{h h h}$ for $p p \rightarrow j j h h h$ using MADGRAPH5_AMCNLO 2.2.3 to generate the events, and ROOT 5.34.25 [36] to obtain the invariant mass distributions. Figures 5 and 6 show the full invariant mass $M_{h h h}$ in TeV for two representative values of $a$ used in Fig. 2. The grey area again marks the region where unitarity is violated.

The invariant mass distributions all appear very similar with respect to $a$ and have their peaks around $1.8 \mathrm{TeV}$ for $\sqrt{s}=13 \mathrm{TeV}, 3.5 \mathrm{TeV}$ for $\sqrt{s}=33 \mathrm{TeV}$ and $7 \mathrm{TeV}$ for $\sqrt{s}=100 \mathrm{TeV}$. However, with increasing $\sqrt{s}$, the distributions smear out and the tail at high $M_{h h h}$ gets longer and flatter reflecting nonunitary behavior of the amplitude with high $M_{h h h}$. Also one can notice how the unitarity bound shifts to higher energies if $a$ approaches 1, as seen in Fig. 2. At $a=0.9$, unitarity violation roughly starts at $M_{h h h}=$ $4 \mathrm{TeV}$ while for $a=0.99$, the unitarity bound is at around

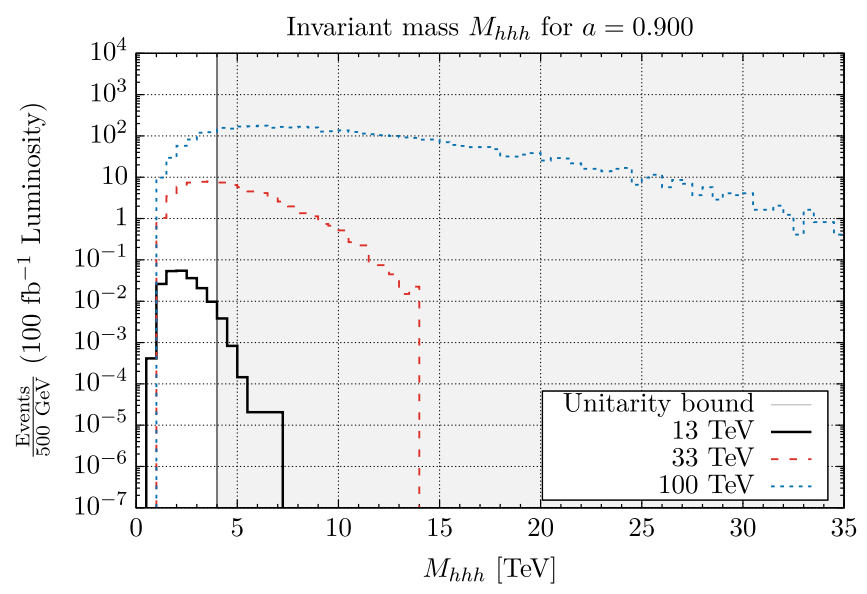

FIG. 5. Invariant mass $M_{h h h}$ in the process $p p \rightarrow j j h h h$ at $a=0.9$ for $\sqrt{s}=13,33,100 \mathrm{TeV}$. The shaded area marks the region where unitarity is violated.

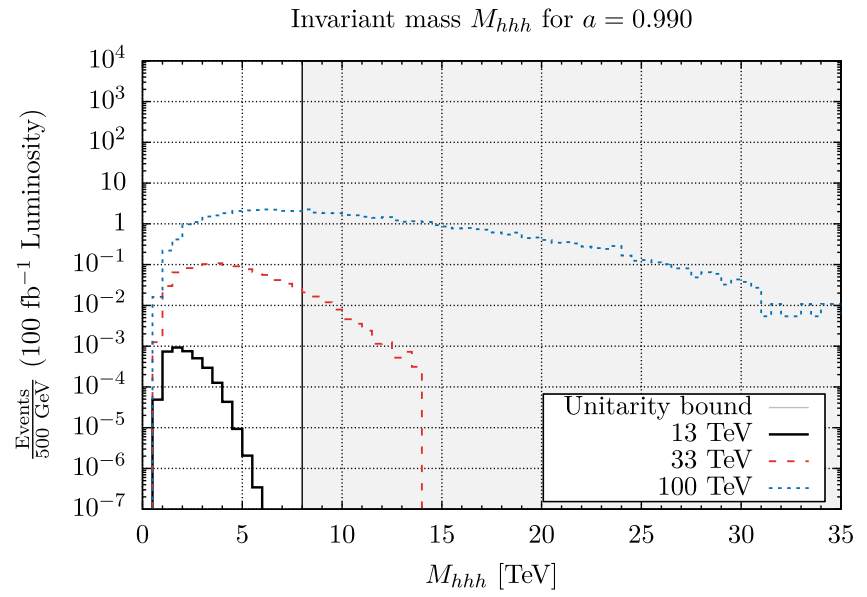

FIG. 6. Invariant mass $M_{h h h}$ in the process $p p \rightarrow j j h h h$ at $a=0.99$ for $\sqrt{s}=13,33,100 \mathrm{TeV}$. The shaded area marks the region where unitarity is violated.
$8 \mathrm{TeV}$. To indicate the proportion of scattering events that violate unitarity for each value of $a$, we define a parameter, $\mathcal{U}$, as:

$$
\mathcal{U}=\frac{\#(\text { events violating unitarity })}{\#(\text { all events })} .
$$

We also define $\varepsilon_{\mathcal{U}}=1-\mathcal{U}$, which gives the proportion of scattering events where unitarity is not violated. Table III shows the fraction of events (or fraction of differential cross section) not violating unitarity with respect to $a$ and $\sqrt{s}$, where we assumed the total integrated luminosity to be $\mathcal{L}_{\text {int }}=100 \mathrm{fb}^{-1}$ for all energies. This allows easy comparisons between the energies and other references.

For $\sqrt{s}=13 \mathrm{TeV}$, unitarity is violated by less than $1 \%$ of scattering events if $a$ is changed by less than $\pm 10 \%$. The total number of events, however, is vanishingly small for the whole range of $a$ and the number of events violating unitarity is even smaller, making it nearly impossible to detect such a signal. For $\sqrt{s}=33 \mathrm{TeV}, \mathcal{U}$ is still below $4 \%$ if $a$ differs only by $1 \%$ from the SM value. Changing $a$ further leads to $\mathcal{U}$ reaching $40 \%$ when $a$ differs by $10 \%$ from the SM. The number of events for $a=1.01$ is about 1 . For $\sqrt{s}=100 \mathrm{TeV}, \mathcal{U}$ is always greater than about $50 \%$ for $1 \%$ deviation from SM and by $85 \%$ for $10 \%$ from the SM. This clearly indicates that the chosen NL $\sigma \mathrm{M}$ cannot be valid at or beyond $\sqrt{s}=100 \mathrm{TeV}$, meaning that new physics should become visible at this energy. The total number of events is comparatively high, ranging from around 50 events for $a=1.01$ to more than 4100 events for $a=0.9$. One can see in case of $100 \mathrm{TeV}$ collider even for $1 \%$ deviation of $h V V$ coupling from the SM one can get a non-negligible number of signal events, however, to judge if the signal can be observed or not we need to estimate the respective background. This is the subject of the next section.

In the end of this section we would like to stress the advantage of $p p \rightarrow j j h h h$ process in comparison to the $p p \rightarrow j j h h$ one, in setting the constraint on $H V V$ coupling down to the permille level - the level which we find in the next section. From Table II one can see that $p p \rightarrow j j h h$ is much weaker enhanced by $\epsilon_{a}=1-a$ then $p p \rightarrow j j h h h$ production in comparison to their SM predictions. For small deviations from SM $\left(\epsilon_{a} \lesssim 0.01\right)$ deviation from SM scales linearly with $\epsilon_{a}$, however this deviation scales as $a E^{4}$ for $p p \rightarrow j j h h h$ while for $p p \rightarrow j j h h$ as $a E$. Using this information or using direct calculation one can estimate that $\sigma(p p \rightarrow j j h h h)$ and $\sigma(p p \rightarrow j j h h)$ will be comparable and of the order of $10^{-3} \mathrm{fb}$ for $\epsilon_{a}=0.01$. At the same time the relative deviation from SM in case of $\sigma(p p \rightarrow$ $j j h h h)$ is much bigger than that of $\sigma(p p \rightarrow j j h h)$ : for $\sigma(p p \rightarrow j j h h)$ process it is only about $16 \%$ for $\epsilon_{a}=0.01$ (and about $1.6 \%$ for $\left.\epsilon_{a}=0.001\right)$, while for $\sigma(p p \rightarrow$ $j j h h h)$ process the respective deviation from SM is factor 1000 for $\epsilon_{a}=0.01$ (and factor 100 for $\epsilon_{a}=0.001$ ). This is 
TABLE III. Proportion of scattering events where unitarity is not violated $\varepsilon_{\mathcal{U}}$ in $\%$ for $M_{h h h}$ with respect to $a$ and $\sqrt{s}$. Also shown are the cross sections $\sigma$ in pb, the total number of events $\mathcal{L}_{\text {int }} \cdot \sigma$ and the proportion of events not violating unitarity $\mathcal{L}_{\text {int }} \cdot \sigma \cdot \varepsilon_{\mathcal{U}}$, where $\mathcal{L}_{\text {int }}$ is the total integrated luminosity. We assume $\mathcal{L}_{\text {int }}=100 \mathrm{fb}^{-1}$ for all energies to allow easy comparisons between the energies.

\begin{tabular}{|c|c|c|c|c|c|c|c|c|c|c|c|c|c|c|}
\hline \multicolumn{5}{|c|}{$13 \mathrm{TeV}$} & \multicolumn{5}{|c|}{$33 \mathrm{TeV}$} & \multicolumn{5}{|c|}{$100 \mathrm{TeV}$} \\
\hline$a$ & $\varepsilon_{\mathcal{U}}[\%]$ & $\sigma[\mathrm{pb}]$ & $\mathcal{L}_{\text {int }} \cdot \sigma$ & $\mathcal{L}_{\text {int }} \cdot \sigma \cdot \varepsilon_{\mathcal{U}}$ & $a$ & $\varepsilon_{\mathcal{U}}[\%]$ & $\sigma[\mathrm{pb}]$ & $\mathcal{L}_{\text {int }} \cdot \sigma$ & $\mathcal{L}_{\text {int }} \cdot \sigma \cdot \varepsilon_{\mathcal{U}}$ & $a$ & $\varepsilon_{\mathcal{U}}[\%]$ & $\sigma[\mathrm{pb}]$ & $\mathcal{L}_{\text {int }} \cdot \sigma$ & $\mathcal{L}_{\text {int }} \cdot \sigma \cdot \varepsilon_{\mathcal{U}}$ \\
\hline 0.80 & 97.81 & $5.70 \times 10^{-6}$ & 0.57 & 0.56 & 0.80 & 44.76 & $2.10 \times 10^{-3}$ & 210.00 & 94.00 & 0.80 & 10.01 & 0.12 & 12000.00 & 1201.20 \\
\hline 0.90 & 99.72 & $2.10 \times 10^{-6}$ & 0.21 & 0.21 & 0.90 & 58.21 & $7.50 \times 10^{-4}$ & 75.00 & 43.66 & 0.90 & 15.38 & $4.10 \times 10^{-2}$ & 4100.00 & 630.58 \\
\hline 0.92 & 99.30 & $1.40 \times 10^{-6}$ & 0.14 & 0.14 & 0.92 & 62.36 & $5.10 \times 10^{-4}$ & 51.00 & 31.80 & 0.92 & 17.48 & $2.80 \times 10^{-2}$ & 2800.00 & 489.44 \\
\hline 0.94 & 99.99 & $8.50 \times 10^{-7}$ & 0.09 & 0.08 & 0.94 & 68.75 & $3.10 \times 10^{-4}$ & 31.00 & 21.31 & 0.94 & 19.84 & $1.70 \times 10^{-2}$ & 1700.00 & 337.28 \\
\hline 0.96 & 100 & $4.10 \times 10^{-7}$ & 0.04 & 0.04 & 0.96 & 76.29 & $1.50 \times 10^{-4}$ & 15.00 & 11.44 & 0.96 & 24.76 & $7.90 \times 10^{-3}$ & 790.00 & 195.60 \\
\hline 0.97 & 100 & $2.40 \times 10^{-7}$ & 0.02 & 0.02 & 0.97 & 82.61 & $8.40 \times 10^{-5}$ & 8.40 & 6.94 & 0.97 & 29.97 & $4.60 \times 10^{-3}$ & 460.00 & 137.86 \\
\hline 0.98 & 100 & $1.10 \times 10^{-7}$ & 0.01 & 0.01 & 0.98 & 89.69 & $3.90 \times 10^{-5}$ & 3.90 & 3.50 & 0.98 & 35.53 & $2.10 \times 10^{-3}$ & 210.00 & 74.61 \\
\hline 0.99 & 100 & $3.40 \times 10^{-8}$ & 0.00 & 0.00 & 0.99 & 96.91 & $1.00 \times 10^{-5}$ & 1.00 & 0.97 & 0.99 & 50.34 & $5.40 \times 10^{-4}$ & 54.00 & 27.18 \\
\hline 1.01 & 100 & $3.60 \times 10^{-8}$ & 0.00 & 0.00 & 1.01 & 96.72 & $1.10 \times 10^{-5}$ & 1.10 & 1.06 & 1.01 & 48.88 & $5.90 \times 10^{-4}$ & 59.00 & 28.84 \\
\hline 1.02 & 100 & $1.30 \times 10^{-7}$ & 0.01 & 0.01 & 1.02 & 88.64 & $4.50 \times 10^{-5}$ & 4.50 & 3.99 & 1.02 & 35.10 & $2.40 \times 10^{-3}$ & 240.00 & 84.24 \\
\hline 1.03 & 100 & $2.90 \times 10^{-7}$ & 0.03 & 0.03 & 1.03 & 81.76 & $1.00 \times 10^{-4}$ & 10.00 & 8.18 & 1.03 & 27.59 & $5.50 \times 10^{-3}$ & 550.00 & 151.75 \\
\hline 1.04 & 99.98 & $5.30 \times 10^{-7}$ & 0.05 & 0.05 & 1.04 & 74.37 & $1.90 \times 10^{-4}$ & 19.00 & 14.13 & 1.04 & 23.16 & $1.00 \times 10^{-2}$ & 1000.00 & 231.60 \\
\hline 1.06 & 99.94 & $1.30 \times 10^{-6}$ & 0.13 & 0.13 & 1.06 & 65.06 & $4.50 \times 10^{-4}$ & 45.00 & 29.28 & 1.06 & 18.07 & $2.40 \times 10^{-2}$ & 2400.00 & 433.68 \\
\hline 1.08 & 99.56 & $2.30 \times 10^{-6}$ & 0.23 & 0.23 & 1.08 & 56.84 & $8.40 \times 10^{-4}$ & 84.00 & 47.75 & 1.08 & 14.94 & $4.50 \times 10^{-2}$ & 4500.00 & 672.30 \\
\hline 1.10 & 99.01 & $3.90 \times 10^{-6}$ & 0.39 & 0.39 & 1.10 & 50.96 & $1.40 \times 10^{-3}$ & 140.00 & 71.34 & 1.10 & 12.12 & $7.50 \times 10^{-2}$ & 7500.00 & 909.00 \\
\hline 1.20 & 91.81 & $2.00 \times 10^{-5}$ & 2.00 & 1.84 & 1.20 & 32.60 & $7.20 \times 10^{-3}$ & 720.00 & 234.72 & 1.20 & 7.04 & 0.39 & 39000.00 & 2745.60 \\
\hline
\end{tabular}

one of the most important points we would like to stress in our paper. Moreover, the respective background for $p p \rightarrow$ $j j h h$ (as we will see below) is at least 3 orders of magnitude higher than the one for $p p \rightarrow j j h h h$. This makes $p p \rightarrow$ $j j h h$ process not to be sensitive to $\epsilon_{a}=0.01$ or below. Even taking into account the fact that $50 \%$ of events will survive after unitarity cut for $p p \rightarrow j j h h h$ process for $\epsilon_{a}=$ 0.01 (for $\epsilon_{a}=0.001$ about $100 \%$ events will survive) the $p p \rightarrow j j h h h$ process plays unique role in probing $\epsilon_{a}$ down to about permille level as we demonstrate below.

\section{ESTIMATION OF BACKGROUND AND COLLIDER SENSITIVITY TO $h V V$ COUPLING}

Triple Higgs production via $\mathrm{VBF}$ gives rise to a spectacular signature at the FCC: the invariant mass of the three Higgs bosons is above several $\mathrm{TeV}$, even for the case when the $h V V$ coupling differs from the SM by only $1 \%$. This makes the Higgs bosons quite boosted and even for $M_{h h h} \simeq 1 \mathrm{TeV}$, which is the lower edge of the $M_{h h h}$ distribution, as one can see from Fig. 6, the cone size around the Higgs boson decay products (e.g., two $b$-jets) will be of the order of $\frac{M_{h}}{2} / \frac{M_{h h h}}{3}=\frac{125 \mathrm{GeV}}{2} / \frac{1000 \mathrm{GeV}}{3} \simeq 0.2$. Therefore, the signature will be two forward-backward jets with a large rapidity gap and three energetic Higgs-jets with a typical radius below 0.2 . In this study, we consider the $h \rightarrow b \bar{b}$ decay channel for all three Higgs bosons. In Ref. [37], the authors have found that the efficiency for the identification of a pair of boosted Higgs bosons from KK-Graviton decays (including $b$-tagging efficiencies) is about $\varepsilon_{h h} \simeq 15 \%$ for Higgs bosons with large enough momentum. These important results are very relevant to our study, where we estimate signal and background rates using this efficiency. We have also checked that these results are in a good agreement with recent analysis performed by ATLAS collaboration [38]. Using $\varepsilon_{h h}$ one can estimate the efficiency for triple Higgs-jet tagging as $\varepsilon_{h h h}=\left(\sqrt{\varepsilon_{h h}}\right)^{3} \simeq 0.058$. Taking into account that $\mathrm{BR}(h \rightarrow b \bar{b}) \simeq 58 \%$, the rate for the tagged triple Higgsjet signature coming from the $p p \rightarrow j j h h h$ VBF process followed by $h \rightarrow b \bar{b}$ decays is given by

$$
\begin{aligned}
\sigma_{\text {sig }}(h h h) & =\sigma(p p \rightarrow j j h h h) \times \varepsilon_{h h h} \times \mathrm{BR}(h \rightarrow b \bar{b})^{3} \\
& \simeq \sigma(p p \rightarrow j j h h h) \times 0.0113
\end{aligned}
$$

We assume that the main background (BG) is coming from the QCD process $p p \rightarrow j j b \bar{b} b \bar{b} b \bar{b}$ ( $6 b \mathrm{BG})$. Before evaluating this process (which is actually not currently possible by means of known matrix-element generators), we have decided to evaluate the $p p \rightarrow b \bar{b} b \bar{b} b \bar{b}$ process to understand the level of the $6 b$-jet background first without the requirement of the two forward-backward jets with large rapidity gap.

To evaluate the background to the triple Higgs-jet signature coming from the $6 b$-jet process, we use a mass window cut

$$
\left|M_{b b}^{i}-M_{h}\right|=\Delta_{M_{h}}^{i} \leq \Delta_{M_{h}}^{\text {cut }}=15 \mathrm{GeV}
$$

for $M_{b b}^{i}(i=1,2,3)$, which represents the three "best" $b b$ or $b \bar{b}$ combinations with the lowest $\Delta_{M_{h}}^{i}$ values. This 
choice allows to avoid combinatorial BG. The choice of $\Delta_{M_{h}}^{\text {cut }}$ (which can be further optimized) is made to be consistent with the jet energy resolution, which is below $10 \%$ at the ATLAS and CMS detectors at the LHC and which is expected to be of the same order at $100 \mathrm{TeV} p p$ FCC's (FCC@100 TeV). We also apply

$$
p_{T}^{b}>50 \mathrm{GeV}, \quad\left|\eta_{b}\right|<2 \text { and } M_{6 b}>1 \mathrm{TeV},
$$

where the first two cuts ensure that the $b$-jets are in the acceptance region and the last one is used to effectively suppress the BG, which drops steeply with $M_{6 b}$, as illustrated below in Fig. 7. At the same time, $M_{6 b}$ for the signal grows with the increase of $M_{6 b}$ for $\varepsilon_{a}=a-1$ in the $10^{-3}-10^{-1}$ range and is not visibly affected by this cut. Besides the above cuts, we also would like to make use of the fact that the Higgs bosons are quite boosted and therefore apply an upper cut on the $\Delta R_{b b}$ separation of the $b$-quarks:

$$
\Delta R_{b b}=\sqrt{\Delta \phi_{b b}+\Delta \eta_{b b}} \leq \Delta R_{b b}^{\mathrm{cut}}=0.5,
$$

which will not affect the signal but will further suppress the BG as we illustrate below in Fig. 7.

There are certain technical problems in the evaluation of $6 b$ BG: the application of the $\Delta_{M_{h}}^{\text {cut }}$ and $M_{6 b}$ cuts at MADGRAPH level in form of user defined cuts lead to zero cross section due to too little phase space left, so MADGRAPH was failing to evaluate it. On the other hand, the $6 b \mathrm{BG}$ was too heavy for the squared matrix element method of CALCHEP to perform the symbolic calculations. However, we still managed to estimate the $6 b$ BG using the following procedure: (a) we evaluated the process $p p \rightarrow$ $b \bar{b} b \bar{b}(4 b$ BG) at parton level using CALCHEP with the cuts given by Eqs. (12)-(14) and simulated the respective events; (b) we have used these events as a user process for PYTHIA 8.2.30 [39] Monte-Carlo generator to find the probability of producing an additional $b \bar{b}$-pair from initial (ISR) and final state radiation (FSR) and have applied the kinematical cuts of equations (12)-(14) on this pair at PYTHIA level; (c) we validated this procedure for lower $b$ quark multiplicities (by simulating the $4 b \mathrm{BG}$ from a $2 b \mathrm{BG}$ starting point) and have found that for ISR/FSR and the QCD scale in PYTHIA chosen to be equal to $\hat{s}$, this estimation works with an accuracy of about 20\%-30\%. One should note that this is sufficient to estimate the $6 \mathrm{~b}$ BG within an order of magnitude as we discuss below. To illustrate the importance of $M_{4 b}$ and $\Delta R_{b b}$ for the $4 b \mathrm{BG}$, we present the following distributions in 7 below: (a) from the left frame, one can see that for the steeply falling $M_{4 b}$ distribution, increasing the $M_{4 b}$ cut from $1 \mathrm{TeV}$ to $1.5 \mathrm{TeV}$ would reduce this BG by about one order of magnitude; (b) from the right frame, one can see that decreasing the upper cut on $\Delta R_{b b}$ (for one of the pairs chosen according to the procedure described above) would also significantly reduce the BG. When applying the cuts (12)-(14), using CTEQ611 as PDF and setting the QCD scale equal to $M_{4 b}$, the cross section (which we then use for the $6 b$ BG estimation) is found to be equal to $19.0 \mathrm{fb}$. As described above, we have used $4 b$ BG events to find the probability $\omega_{b b}$ to create an additional $b \bar{b}$ pair with $\left|M_{b b}-M_{h}\right| \leq$ $\Delta_{M_{h}}^{\text {cut }}=15 \mathrm{GeV}$ for various values of the $\Delta R_{b b}^{\text {cut }}$ cut. After running $500 \mathrm{~K}$ events through PYTHIA, the respective error for $\omega_{b b}$ lies at the percent level. The results are presented in Table IV below and one can see right away that the cut on
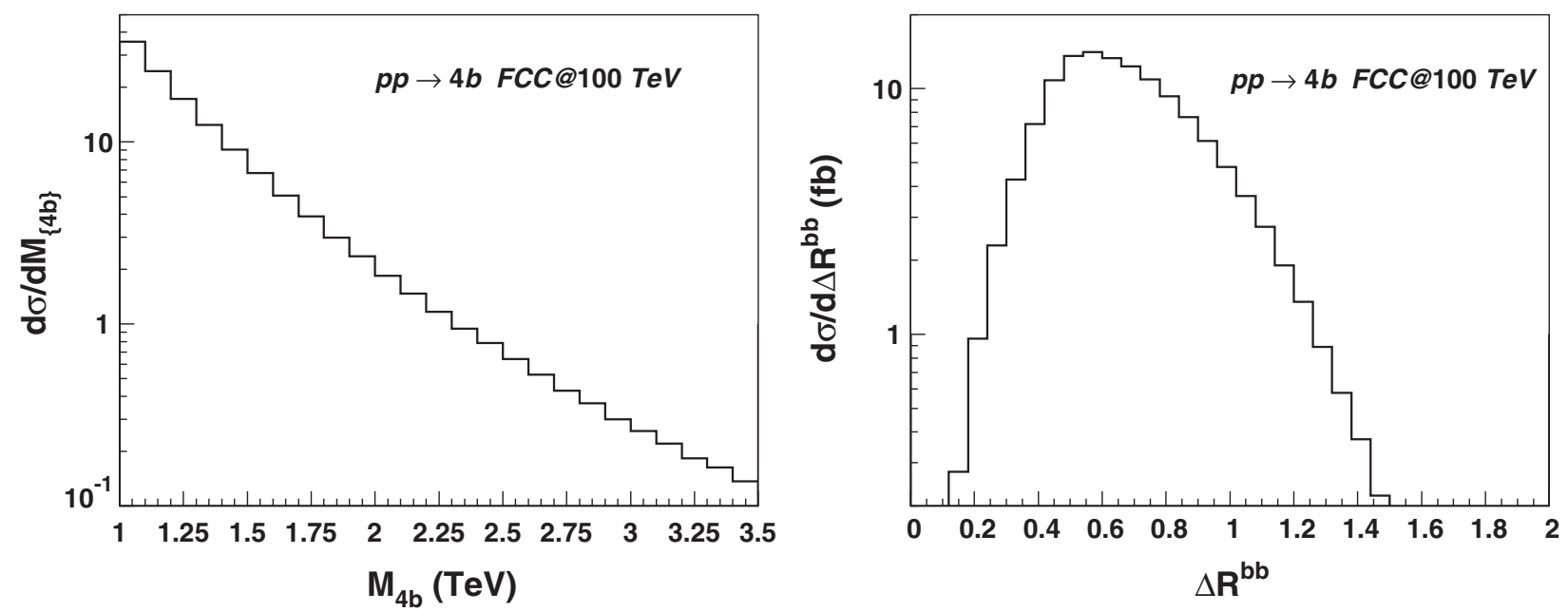

FIG. 7. Distributions of $M_{4 b}$ (left) and $\Delta R_{b b}$ (right) for the process $p p \rightarrow b \bar{b} b \bar{b}$ used for the $6 b$ BG generation via PYTHIA for the cuts (12)-(13) applied at parton level. 
TABLE IV. Probability $\omega_{b b}$ to create an additional $b \bar{b}$ pair from $4 b$ events with $\left|M_{b b}-M_{h}\right| \leq \Delta_{M_{h}}^{\text {cut }}=15 \mathrm{GeV}$ for various values of $\Delta R_{b b}^{\text {cut }}$ cut as a result of running $500 \mathrm{k} 4 b$ events through PYTHIA.

\begin{tabular}{lcccc}
\hline \hline$\Delta R_{b b}<\Delta R_{b b}^{\text {cut }}$ & 2.0 & 1.5 & 1.0 & 0.5 \\
\hline$\omega_{b b}$ & $1.1 \times 10^{-3}$ & $7.0 \times 10^{-4}$ & $3.5 \times 10^{-4}$ & $8.6 \times 10^{-5}$ \\
\hline \hline
\end{tabular}

$\Delta R_{b b}$ has the power to further reduce the SM BG. For $\Delta R_{b b}<0.5, \omega_{b b}=8.6 \times 10^{-5}$ and $\sigma(6 \mathrm{~b})$ for the cuts (12)(14) can be estimated as:

$$
\begin{aligned}
\sigma(6 \mathrm{~b}) & =\sigma(4 \mathrm{~b}) \times \omega_{b b}\left(\Delta R_{b b}<0.5\right) \\
& =19.0 \mathrm{fb} \times 8.6 \times 10^{-5} \\
& \simeq 1.6 \times 10^{-3} \mathrm{fb} .
\end{aligned}
$$

After the procedure of triple Higgs-jet tagging, the rate of the hhh BG can be estimated as

$$
\begin{aligned}
\sigma_{B G}(\mathrm{hhh}) & =\sigma(6 b) \times \varepsilon_{h h h}, \\
& \simeq 9.5 \times 10^{-5} \mathrm{fb}
\end{aligned}
$$

while the signal rate is given by Eq. (11).

One can check from Eq. (16) of Ref. [22] that $\sigma(p p \rightarrow j j h h h)$ quite precisely scales as $\varepsilon_{a}^{2}=(1-a)^{2}$, when $\left|\varepsilon_{a}\right| \ll 1$ and $\sigma(p p \rightarrow j j h h h) \gg \sigma(p p \rightarrow j j h h h)_{\mathrm{SM}}$. Using this scaling and the rates from Table III one can easily find the signal rates for smaller values of $\varepsilon_{a}$. In Fig. 8 we present, $\sigma_{s i g}(h h h)$ and $\sigma_{\mathrm{BG}}(h h h)$ for $\varepsilon_{a}$ in the range [-0.01:0.01] (left frame) as well as the $100 \mathrm{TeV}$ FCC sensitivity to $\varepsilon_{a}$ (right frame). One can see that the signal dominates over the $6 b \mathrm{BG}$ and becomes comparable to the $6 b$ BG only for $\left|\varepsilon_{a}\right|$ at the permille level or below. The dotted curves in both frames present results for the signal equal to $\sigma_{\text {sig }}(h h h) \times \varepsilon_{\mathcal{U}}$ to take into account the cut of the region of the parameter space where unitarity is violated.

One should note, that our BG estimation should be considered as an upper bound for the BG, since after the requirement of two additional forward-backward jets, the actual BG is expected to be two orders of magnitude below just the $6 b$ BG. Therefore, we can safely assume that for $\left|\varepsilon_{a}\right|>10^{-3}$, the actual BG is negligible in comparison to the signal, hence it is only a question of luminosity to probe $\varepsilon_{a}$ up to the permille level. For example, with $100 \mathrm{fb}^{-1}$, $1 \mathrm{ab}^{-1}$ and $10 \mathrm{ab}^{-1}$, one can probe $\left|\varepsilon_{a}\right| \simeq 2.5 \times 10^{-2},\left|\varepsilon_{a}\right| \simeq$ $7.5 \times 10^{-3}$ and $\left|\varepsilon_{a}\right| \simeq 2.5 \times 10^{-3}$ respectively. We have used two standard deviations criteria to judge about this sensitivity, which is indicated in the right pane of Fig. 8 together with the $5 \sigma$ discovery limit in form of two horizontal lines at 2 and 5 respectively. Altogether, one can see that with triple Higgs VBF signatures at a $100 \mathrm{TeV}$ FCC, we will be able to measure the $h V V$ coupling with permille accuracy. This accuracy is remarkable since it is about two orders of magnitude better than the sensitivity achievable at the LHC.

It is important to stress separately findings of [40] (see also [41] in this relation) where authors has suggested to measure $h h V V$ coupling ( $b$ coupling from Eq. (8) in Higgs pair production using also VBF production process and boosted Higgs technique. It was found in [40] that this process can have few fb production cross section for about $10 \% h h V V$ deviation from SM and that with $10 \mathrm{ab}^{-1} h h V V$ can be measured with about $1 \%$ precision. This is an impressive and complementary result to our study.
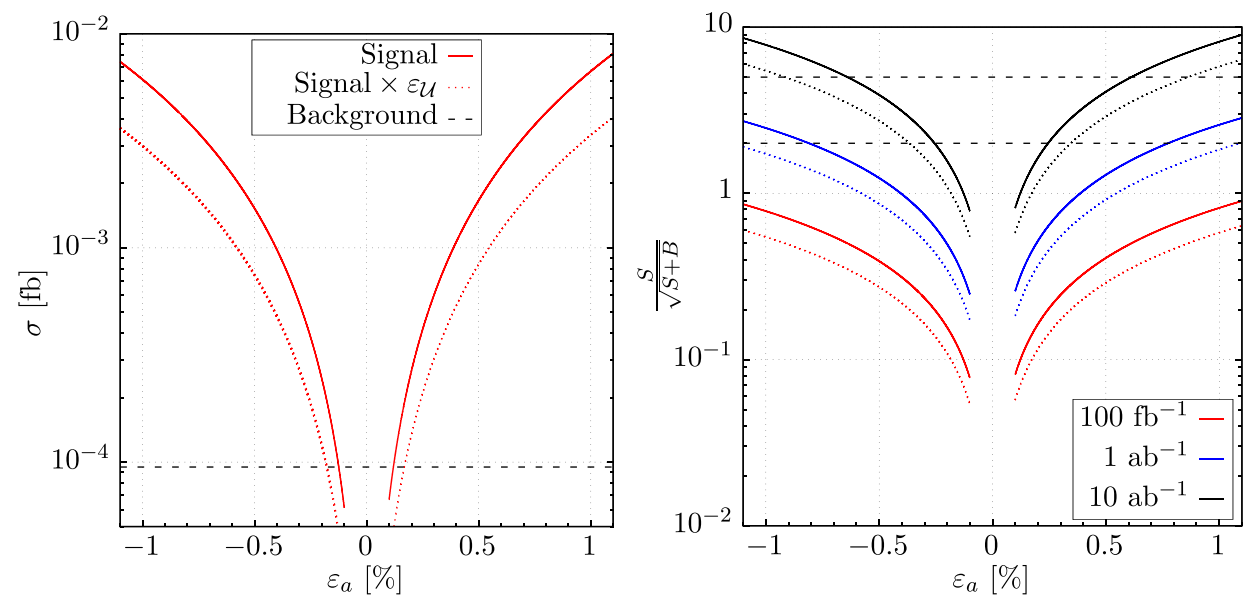

FIG. 8. $\sigma_{\mathrm{sig}}(h h h)$ and $\sigma_{\mathrm{BG}}(h h h)$ for $\varepsilon_{a} \in[-0.01: 0.01]$ (left frame) as well as the $100 \mathrm{TeV}$ FCC sensitivity to $\varepsilon_{a}$ (right frame) for $100 \mathrm{fb}^{-1}, 1 \mathrm{ab}^{-1}$ and $10 \mathrm{ab}^{-1}$ integrated luminosities benchmarks. The dotted curves in both frames present results for the signal equal to $\sigma_{\text {sig }}(h h h) \times \varepsilon_{\mathcal{U}}$. 


\section{CONCLUSIONS}

We have explored the potential of future hadron colliders to test the couplings of a Higgs boson to gauge bosons. As has been shown previously, if the coupling of the Higgs boson to gauge bosons deviates from the standard model, multiboson production via vector-boson scattering can be hugely enhanced in comparison to the SM due to the lack of cancellation in longitudinal vector boson scattering. Among these processes, triple Higgs boson production plays a special role-its enhancement is especially spectacular due to the absence of background from transversely polarized vector bosons in the final state. While the rates from $p p \rightarrow j j h h h$ production in vector boson fusion are too low at the LHC and even at future $33 \mathrm{TeV} p p$ colliders, we have found that the $100 \mathrm{TeV} p p \mathrm{FCC}$ has the unique opportunity to probe the $h V V$ coupling far beyond the LHC sensitivity using triple Higgs production via vector boson fusion.

We have evaluated the $p p \rightarrow j j h h h$ rates as a function of the deviation from the $h V V$ coupling, $\varepsilon_{a}$, before and after VBF cuts and have estimated the $6 b$-jet backgroundwhich turns out to be much smaller than the signal for $\left|\varepsilon_{a}\right|>10^{-3}$-and have found that the $100 \mathrm{TeV} p p$ FCC can probe this coupling with high precision. A summary of our findings is presented in Fig. 8, demonstrating the impressive sensitivity to the $h V V$ coupling of the $100 \mathrm{TeV}$ $p p$ FCC via $h h h$ production in vector boson fusion up to permille accuracy. This sensitivity, which is about two orders of magnitude better than the sensitivity reachable at the LHC, highlights a special role of the hhh VBF production and stresses once more the importance of the $100 \mathrm{TeV} p p$ FCC.

As final remark we note that we chose the effective theory described by Eq. (8) with only one coupling a deviating from the SM one. In more generic scenarios one could expect the whole set of couplings to differ from the SM one, like for example in minimal composite Higgs model [42] (MCHM4), however the generic effect of VBF hhh enhancement as well as its value is similar to the scenario we study here as it was shown in [22]. The nonunitary behavior of the VBF cross section will be eventually restored in the complete theory with, for example, resonances at some higher scale. However if this scale is sufficiently high, the enhancement of VBF hhh production can be quite dramatic and $100 \mathrm{TeV} p p$ FCC would play a very important role to test such a scenario and related couplings to a very high level of precision with this process.

\section{ACKNOWLEDGMENTS}

The authors acknowledge the use of the IRIDIS High Performance Computing Facility, and associated support services at the University of Southampton, in the completion of this work. A. B. would like to thank Douglas Ross for valuable discussions on the estimation of the QCD background. A. B. and P. B. S. also grateful to Micheleangelo Mangano for various discussions and help with ALPGEN package, Alexandra Carvalho for discussion and help with understanding efficiencies for Higgs-jet tagging. A. B. and P. B. S. acknowledge partial support from the InvisiblesPlus RISE from the European Union Horizon 2020 research and innovation programme under the Marie Sklodowska-Curie Grant Agreement No. 690575. A. B. acknowledges partial support from the STFC Grant No. ST/L000296/1. A. B. also thanks the NExT Institute, Royal Society Leverhulme Trust Senior Research Fellowship LT140094, Royal Society Internationl Exchange Grant No. IE150682 and SotonFAPESP grant. A. B. also acknowledge the support of IBS centre in Daejeon for the hospitality and support.
[1] G. Aad et al. (ATLAS Collaboration), Observation of a new particle in the search for the Standard Model Higgs boson with the ATLAS detector at the LHC, Phys. Lett. B 716, 1 (2012).

[2] S. Chatrchyan et al. (CMS Collaboration), Observation of a new boson at a mass of $125 \mathrm{GeV}$ with the CMS experiment at the LHC, Phys. Lett. B 716, 30 (2012).

[3] ATLAS Collaboration, Combined measurements of Higgs boson production and decay using up to $80 \mathrm{fb}^{-1}$ of protonproton collision data at $\sqrt{s}=13 \mathrm{TeV}$ collected with the ATLAS experiment (2018).

[4] CMS Collaboration, Combined measurements of the Higgs boson's couplings at $\sqrt{s}=13 \mathrm{TeV}$ (2018).

[5] CMS Collaboration, Sensitivity projections for Higgs boson properties measurements at the HL-LHC (2018).
[6] ATLAS Collaboration, Physics at a high-luminosity LHC with ATLAS, in Proceedings of the 2013 Community Summer Study on the Future of U.S. Particle Physics: Snowmass on the Mississippi (CSS2013): Minneapolis, MN, USA, 2013 (2013), http://www.slac.stanford.edu/econf/ C1307292/docs/submittedArxivFiles/1307.7292.pdf.

[7] X.-G. He, G.-N. Li, and Y.-J. Zheng, Probing Higgs boson $C P$ properties with $t \bar{t} H$ at the LHC and the $100 \mathrm{TeV} p p$ collider, Int. J. Mod. Phys. A 30, 1550156 (2015).

[8] A. J. Barr, M. J. Dolan, C. Englert, D. E. Ferreira de Lima, and M. Spannowsky, Higgs self-coupling measurements at a $100 \mathrm{TeV}$ hadron collider, J. High Energy Phys. 02 (2015) 016.

[9] M. Low and L.-T. Wang, Neutralino dark matter at $14 \mathrm{TeV}$ and 100 TeV, J. High Energy Phys. 08 (2014) 161. 
[10] B. S. Acharya, K. Bożek, C. Pongkitivanichkul, and K. Sakurai, Prospects for observing charginos and neutralinos at a $100 \mathrm{TeV}$ proton-proton collider, J. High Energy Phys. 02 (2015) 181.

[11] B. Auerbach, S. Chekanov, J. Love, J. Proudfoot, and A. Kotwal, Sensitivity to new high-mass states decaying to $t \bar{t}$ at a 100 TeV collider, Phys. Rev. D 91, 034014 (2015).

[12] A. Fowlie and M. Raidal, Prospects for constrained supersymmetry at $\sqrt{s}=33 \mathrm{TeV}$ and $\sqrt{s}=100 \mathrm{TeV}$ protonproton super-colliders, Eur. Phys. J. C 74, 2948 (2014).

[13] N. Arkani-Hamed, T. Han, M. Mangano, and L.-T. Wang, Physics opportunities of a $100 \mathrm{TeV}$ proton-proton collider, Phys. Rep. 652, 1 (2016).

[14] J. Baglio, A. Djouadi, and J. Quevillon, Prospects for Higgs physics at energies up to $100 \mathrm{TeV}$, Rep. Prog. Phys. 79, 116201 (2016).

[15] M. Bicer et al. (TLEP Design Study Working Group), First look at the physics case of TLEP, J. High Energy Phys. 01 (2014) 164.

[16] S. Dawson et al., Working group report: Higgs boson, in Proceedings of the 2013 Community Summer Study on the Future of U.S. Particle Physics: Snowmass on the Mississippi (CSS2013): Minneapolis, MN, USA, 2013 (2013).

[17] R. Contino et al., Physics at a $100 \mathrm{TeV}$ pp collider: Higgs and EW symmetry breaking studies, CERN Yellow Report No. CERN-TH-2016-113, 2017.

[18] D. M. Asner et al., ILC Higgs white paper, in Proceedings of the 2013 Community Summer Study on the Future of U.S. Particle Physics: Snowmass on the Mississippi (CSS2013): Minneapolis, MN, USA, 2013 (2013), http://www.slac .stanford.edu/econf/C1307292/docs/submittedArxivFiles/ 1310.0763.pdf.

[19] B. Fuks, J. H. Kim, and S. J. Lee, Scrutinizing the Higgs quartic coupling at a future $100 \mathrm{TeV}$ proton-proton collider with taus and b-jets, Phys. Lett. B 771, 354 (2017).

[20] M. Mangano, Physics at the FCC-hh, a $100 \mathrm{TeV}$ pp collider, CERN Yellow Report No. CERN 2017-003-M.

[21] S. Borowka, C. Duhr, F. Maltoni, D. Pagani, A. Shivaji, and $\mathrm{X}$. Zhao, Probing the scalar potential via double Higgs boson production at hadron colliders, arXiv:1811.12366 [J. High Energy Phys. (to be published)].

[22] A. Belyaev, A. Oliveira, R. Rosenfeld, and M. C. Thomas, Multi Higgs and vector boson production beyond the standard model, J. High Energy Phys. 05 (2013) 005.

[23] D. A. Dicus and H.-J. He, Scales of fermion mass generation and electroweak symmetry breaking, Phys. Rev. D 71, 093009 (2005).

[24] F. Maltoni, J. Niczyporuk, and S. Willenbrock, The scale of fermion mass generation, Phys. Rev. D 65, 033004 (2002).

[25] E. Byckling and K. Kajantie, Particle Kinematics (John Wiley \& Sons, New York, 1973).

[26] J. M. Cornwall, D. N. Levin, and G. Tiktopoulos, Derivation of gauge invariance from high-energy unitarity bounds on the S matrix, Phys. Rev. D 10, 1145 (1974); Phys. Rev. D 11, 972(E) (1975).

[27] B. W. Lee, C. Quigg, and H. B. Thacker, Weak interactions at very high-energies: The role of the Higgs boson mass, Phys. Rev. D 16, 1519 (1977).

[28] M. S. Chanowitz and M. K. Gaillard, The TeV physics of strongly interacting W's and Z's, Nucl. Phys. B261, 379 (1985).

[29] S. S. D. Willenbrock, Pair production of $W$ and $Z$ bosons and the Goldstone boson equivalence theorem, Ann. Phys. (N.Y.) 186, 15 (1988).

[30] J. Bagger and C. Schmidt, Equivalence theorem Redux, Phys. Rev. D 41, 264 (1990).

[31] H. G. J. Veltman, The equivalence theorem, Phys. Rev. D 41, 2294 (1990).

[32] G. Giudice, C. Grojean, A. Pomarol, and R. Rattazzi, The strongly-interacting light Higgs, J. High Energy Phys. 06 (2007) 045.

[33] J. Alwall, R. Frederix, S. Frixione, V. Hirschi, F. Maltoni, O. Mattelaer, H.-S. Shao, T. Stelzer, P. Torrielli, and M. Zaro, The automated computation of tree-level and next-toleading order differential cross sections, and their matching to parton shower simulations, J. High Energy Phys. 07 (2014) 079.

[34] J. Pumplin, D. R. Stump, J. Huston, H.-L. Lai, P. Nadolsky, and W.-K. Tung, New generation of parton distributions with uncertainties from global QCD analysis, J. High Energy Phys. 07 (2002) 012.

[35] A. Belyaev, N. D. Christensen, and A. Pukhov, CalcHEP 3.4 for collider physics within and beyond the Standard Model, Comput. Phys. Commun. 184, 1729 (2013).

[36] R. Brun and F. Rademakers, ROOT-An object oriented data analysis framework, Nucl. Instrum. Methods Phys. Res., Sect. A 389, 81 (1997).

[37] M. Gouzevitch, A. Oliveira, J. Rojo, R. Rosenfeld, G. P. Salam, and V. Sanz, Scale-invariant resonance tagging in multijet events and new physics in Higgs pair production, J. High Energy Phys. 07 (2013) 148.

[38] M. Aaboud et al. (ATLAS Collaboration), Search for pair production of Higgs bosons in the $b \bar{b} b \bar{b}$ final state using proton-proton collisions at $\sqrt{s}=13 \mathrm{TeV}$ with the ATLAS detector, arXiv:1804.06174.

[39] T. Sjostrand, S. Mrenna, and P.Z. Skands, A brief introduction to PYTHIA 8.1, Comput. Phys. Commun. 178, 852 (2008).

[40] F. Bishara, R. Contino, and J. Rojo, Higgs pair production in vector-boson fusion at the LHC and beyond, Eur. Phys. J. C 77, 481 (2017).

[41] C. Englert, Q. Li, M. Spannowsky, M. Wang, and L. Wang, VBS $W^{ \pm} W^{ \pm} H$ production at the HL-LHC and a $100 \mathrm{TeV}$ pp-collider, Int. J. Mod. Phys. A 32, 1750106 (2017).

[42] R. Contino, D. Marzocca, D. Pappadopulo, and R. Rattazzi, On the effect of resonances in composite Higgs phenomenology, J. High Energy Phys. 10 (2011) 081. 